\title{
Epidemiologia de Lipeurus caponis (Phthiraptera: Philopteridae) (Nitzsch, 1818) em granjas avícolas comerciais de postura no estado de Minas Gerais, Brasil*
}

\section{Epidemiology of Lipeurus caponis (Phthiraptera: Philopteridae) (Nitzsch, 1818) in commercial laying hen flocks from the State of Minas Gerais, Brazil}

\author{
Leandro do Carmo Rezende, ${ }^{* *}$ Lucas Maciel Cunha, ${ }^{* * * * *}$ Nelson Rodrigo da Silva Martins, ${ }^{* *}$ Cristina Mara Teixeira, ${ }^{* *}$ \\ Paulo Roberto de Oliveira**†
}

\begin{abstract}
Resumo
Aspectos relacionados com a epidemiologia de Lipeurus caponis foram estudados com o objetivo de determinar os principais fatores de risco para a ocorrência deste ectoparasito em granjas avícolas de postura no estado de Minas Gerais, Brasil. Quatrocentos e trinta e um galpões de granjas de postura comerciais foram visitados no ano de 2012 para a coleta de amostras de ectoparasitos. Também foi aplicado um questionário sobre características ambientais e de manejo utilizado nos galpões de criação das aves. L. caponis foi encontrado em quatro dos 431 galpões estudados $(0,92 \%)$. Os principais determinantes epidemiológicos foram investigados utilizando-se testes de Qui-quadrado, testes exatos de Fisher e modelos multivariados de regressão de Poisson. $\mathrm{O}$ tipo de instalação obteve uma Razão de Prevalência igual a 51,56, o que sugere que aves criadas sobre o solo possuem maior risco de infestação por $L$. caponis do que aves mantidas em gaiolas. Também foi verificado que a linhagem genética das galinhas foi um fator de risco e, provavelmente, algumas linhagens são mais predispostas a infestações. Além disso, a presença de criações de subsistência de galinhas domésticas nas adjacências dos galpões avícolas aumentou consideravelmente o risco de ocorrência de infestações por $L$. caponis nas galinhas poedeiras de granjas comerciais no estado de Minas Gerais.
\end{abstract}

Palavras-chave: Ischnocera, Mallophaga, piolho da asa de galinhas, piolho de galinhas.

\begin{abstract}
Aspects related to the epidemiology of Lipeurus caponis were studied in order to determine the main risk factors for the occurrence of this ectoparasite in commercial laying hen flocks in the State of Minas Gerais, Brazil. An observational, analytical and sectional study was conducted for verifying these factors. Four hundred thirty-one laying poultry flocks were visited in 2012 year to ectoparasite sampling collection and application of a questionnaire about environmental and management characteristics in chicken houses. $L$. caponis was found in four of the 431 studied chicken houses $(0.92 \%)$. The main epidemiological determinants for the occurrence of $L$. caponis were investigated using Chi-square Test, Fisher's Exact Test and multivariate Poisson regression models. Type of facilities obtained an Incidence Rate Ratio (IRR) equal to 51.56, suggesting that poultry bred on the ground have a higher risk of infestation by $L$. caponis than fowls kept in cages. Also it was observed that chicken's genetic lineage was a marginal risk factor $(I R R=1.8)$ and probably some lineages are more predisposed to infestations. Moreover, the presence of subsistence breeding of domestic chickens around the chicken houses (IRR=106.89) increases extensively the risk of occurrence of infestations by $L$. caponis in commercial laying hen flocks from the State of Minas Gerais.
\end{abstract}

Keywords: Chicken lice, Ischnocera, Mallophaga, Poultry wing louse.

\section{Introdução}

Os malófagos são invertebrados pertencentes à classe Insecta e ordem Phthiraptera, popularmente conhecidos como "piolhos". Desses, Lipeurus caponis (Nitzsch, 1818), também conhecido como piolho da asa, pertence à subordem Ischnocera e à família Philopteridae (Guimarães et al., 2001). O hospedeiro primário é a galinha doméstica (Gallus gallus domesticus), embora existam relatos de sua ocorrência em galiformes selvagens mantidos em zoológicos. Trata-se de uma espécie geralmente de pequena atividade e que possui baixo efeito patogênico para as aves (Emerson, 1956). Galinhas são afetadas por este parasito somente quando estão altamente infestadas, o que aparentemente é raro (Price e Graham, 1997).

No Brasil, a presença de $L$. caponis já foi relatada tanto em plantéis comerciais quanto em criações de subsistência (Guimarães et al., 2001). Em 1993 foi realizado um levantamento de malófagos em quarenta e quatro estabelecimentos de criação de galinhas nos estados de São Paulo, Pernambuco,

\footnotetext{
${ }^{*}$ Recebido em 29 de julho de 2014 e aceito em 14 de janeiro de 2015.

**Universidade Federal de Minas Gerais, Escola de Veterinária, Departamento de Medicina Veterinária Preventiva, Belo Horizonte, Minas Gerais. Avenida Presidente Antônio Carlos 6627, Caixa Postal 567, CEP 30123-970, Campus da UFMG, Belo Horizonte - Minas Gerais, Brasil.

***Universidade Federal de Ouro Preto, Instituto de Ciências Exatas e Biológicas, Centro de Ciência Animal. Campus Universitário Morro do Cruzeiro, Ouro

Preto, Minas Gerais, CEP 35400-000, Ouro Preto - Minas Gerais, Brasil. Autor para correspondência: lucasmcunha82@gmail.com.

† In memoriam.
} 
Bahia, Alagoas e Paraná (Figueiredo et al., 1993). A presença de $L$. caponis foi verificada em baixa frequência e somente em criações denominadas pelos autores como "terreiro". Em um levantamento realizado em criações de subsistência no estado de Minas Gerais encontraram-se galinhas domésticas naturalmente infestadas por $L$. caponis (Santos-Prezoto et al., 2003). Em estudo feito em galinhas caipiras no Maranhão, $L$. caponis foi uma das espécies de fitirápteros mais frequentes (Guerra et al., 2008). No estado do Rio Grande do Sul, em criações coloniais de galinhas, a ocorrência estimada dessa espécie foi de 3,0\% (Santos et al., 2013).

Embora existam muitos relatos da ocorrência de malófagos nas criações avícolas, são poucos os estudos acerca dos aspectos epidemiológicos das infestações de L. caponis em Gallus gallus domesticus. Em um estudo realizado no Paquistão com diversas espécies de fitirápteros, observou-se que a ocorrência destes parasitos é maior em criações com altas densidades de galinhas e em aves mais velhas, com idade entre 36 e 72 semanas (Nadeem et al., 2007). Em outro estudo realizado em diversos estados do Brasil, constatou-se que L. caponis e outras espécies de Ischnocera são mais frequentes em aves de criações de subsistência do que em granjas comerciais devido ao hábito destas espécies de viverem mais sobre as penas do que sobre a pele e, consequentemente, à maior dependência da luz solar para sobrevivência. Assim, em criações de subsistência, onde as aves são mantidas em locais com maior exposição à luz solar, a ocorrência destas espécies seria maior (Figueiredo et al., 1993). Ainda segundo este estudo, para o controle das infestações por fitirápteros em granjas avícolas recomenda-se o monitoramento constante das aves, o tratamento individual daquelas parasitadas com sílica aerogel e, no caso de granjas intensamente infestadas, a pulverização de inseticida por alta pressão. Este estudo tem o objetivo de quantificar a ocorrência desse fitiráptero em granjas avícolas de postura comercial no estado de Minas Gerais e verificar fatores de risco para a ocorrência de infestações.

\section{Material e métodos}

O presente estudo foi realizado em granjas avícolas comerciais de postura no estado de Minas Gerais. Durante a visita às propriedades, a temperatura e a umidade dos respectivos municípios foram monitoradas para verificar se variações climáticas poderiam afetar a ocorrência dos ectoparasitos nas granjas. Não foram visitadas propriedades em cujas cidades foram verificadas temperaturas inferiores $5^{\circ} \mathrm{C}$ ou superiores a $40^{\circ} \mathrm{C}$.

A seleção das granjas e a estratificação das amostras foram realizadas considerando-se a proporção de granjas avícolas comerciais de postura em cada uma das 20 regiões administrativas do Instituto Mineiro de Agropecuária (IMA). Além disso, para o cálculo do número de granjas a serem visitadas, foi considerada uma prevalência hipotética de $50 \%$, erro padrão de $5 \%$ e um número finito de propriedades. Assim, de março a julho do ano de 2012, 431 galpões de 43 granjas foram visitados.

Em cada galpão foram colhidas amostras de esterco para a pesquisa de artrópodes associados ao ambiente avícola. Além disso, procedeu-se à inspeção visual de cerca de 20 aves por galpão para coleta de penas e ectoparasitos. Quando à inspeção era percebida a presença de $L$. caponis, utilizava-se fita adesiva para a captura de espécimes (Figueiredo et al., 1993). As amostras foram embaladas em sacos plásticos, os quais foram transportados para o Laboratório de Doenças Parasitárias da Escola de Veterinária da Universidade Federal de Minas Gerais, onde foram mantidas refrigeradas $\left(-20 \pm 2{ }^{\circ} \mathrm{C}\right)$.

Os espécimes de $L$. caponis e outros malófagos encontrados foram clarificados em Lactofenol (Fenol 20g, Ácido lático 16ml, Glicerol 32ml, Água destilada 20ml; Vetec Química Fina Ltda., Rio de Janeiro, Brasil) por 24 horas e posteriormente montados entre lâmina e lamínula (Krantz, 1978). Em seguida, foi realizada a identificação dos artrópodes e ectoparasitos em microscópio estereoscópico (Olympus C35 AD-4) conforme características morfológicas indicadas em literatura específica (Emerson, 1956; Price e Graham, 1997; Guimarães et al., 2001).

Durante as visitas às granjas, o questionário de Cunha (2013), com adaptações, foi aplicado com o objetivo de se obter informações relacionadas aos fatores predisponentes para a ocorrência de ectoparasitos. Uma versão preliminar desse instrumento de coleta de dados contendo 97 alternativas de questões objetivas ou categóricas foi preparada e submetida a um procedimento de validação teste-reteste, utilizando-se o coeficiente Kappa. A versão final do questionário continha 89 alternativas e foi utilizada em entrevistas com proprietários ou funcionários das granjas visitadas. Este estudo foi aprovado pelos Comitês de Ética da Universidade Federal de Minas Gerais (COEP/UFMG número 0238.0.203.000-11 e CETEA/ UFMG 41/2011).

A avaliação dos fatores de risco para a ocorrência de infestações por $L$. caponis foi realizada através do software Stata ${ }^{\circledR} 12.0$, utilizando-se modelos multivariados de regressão de Poisson, conforme descrito por Dohoo et al. (2003). A unidade de análise estatística consistiu nos galpões das granjas, o que levou à realização de compilação da presença ou ausência de $L$. caponis e das características avaliadas no questionário em cada galpão visitado no estudo. A seleção preliminar das variáveis a serem incluídas nos modelos de regressão de Poisson foi realizada utilizando-se o teste de qui-quadrado, teste exato de Fisher e regressão logística univariada, removendo variáveis com Valor-p menor que 0,15 . As variáveis selecionadas na triagem foram utilizadas em modelos de regressão de Poisson, sendo excluídas aquelas não significativas ( $p \geq 0.05$ ) ou que atuassem como variável de confundimento. Aanálise do intervalo de confiança da Razão de Prevalência (em inglês "Incidence Rate Ratio - IRR") com $95 \%$ de confiança também foi utilizada na seleção das variáveis e construção dos modelos. Variáveis com Razão de Prevalência significativas ( $p \leq 0.05$ ) foram mantidas no modelo definitivo de regressão de Poisson. $O$ teste de qui-quadrado de Pearson foi utilizado para a verificação da qualidade do ajuste do modelo $(p>0,05)$.

\section{Resultados e discussão}

A presença de $L$. caponis foi verificada em quatro dos 431 galpões visitados $(0,92 \%)$. O ajuste adequado de parte das variáveis em modelos de regressão com distribuição de Poisson se deveu à baixa frequência de ocorrência dessa espécie de fitiráptero. Esses resultados acerca da frequência de ocorrência concordam com outros trabalhos, nos quais essa espécie apresentou taxas de prevalência relativamente menores. Esse é o caso de um levantamento realizado em quarenta e quatro 
estabelecimentos de criação de galinhas nos estados de São Paulo, Pernambuco, Bahia, Alagoas e Paraná, abrangendo estabelecimentos comerciais e de subsistência (Figueiredo et al., 1993). Segundo esse estudo, L. caponis também ocorreu em baixa frequência, tendo sido observada sua presença somente em tipos de criações em "terreiro", termo que se refere às criações de avicultura de subsistência sob o piso e geralmente não pertencentes às linhagens genéticas comerciais utilizadas na avicultura comercial.

Segundo Figueiredo et al. (1993), a maior ocorrência de $L$. caponis e de outras espécies da subordem Ischnocera em aves de criações de subsistência seria atribuída ao hábito destas espécies de viverem mais sobre as penas do que sobre a pele. Consequentemente, tais espécies teriam maior dependência da luz solar para sobrevivência. Assim, em granjas comerciais, onde as aves são mantidas em galpões sombreados, a tendência seria uma menor ocorrência destes malófagos. Em criações de subsistência, onde as aves seriam mantidas em locais com maior exposição à luz solar, a ocorrência destas espécies seria maior, assim como foi observado no presente estudo.

Em um levantamento realizado no estado de Minas Gerais, também foram encontrados indivíduos de Gallus gallus domesticus mantidos em criações de subsistência naturalmente infestados por $L$. caponis assim como em um estudo em criações de terreiro na República Tcheca (Santos-Prezoto et al., 2003; Sychra et al., 2008). Também em criações de galinhas "caipiras" no estado do Maranhão foi observada a presença de L. caponis (Guerra et al., 2008). L. caponis foi o malógafo mais prevalente em granjas avícolas comerciais no Paquistão (Nadeem et al., 2007). Em outro estudo, realizado no estado da Paraíba, a prevalência de $L$. caponis em galinhas de subsistência foi a segunda maior entre as espécies de Phthiraptera, tanto em ambientes em que as galinhas eram mantidas sem o contato com outras espécies de aves quanto naqueles que eram mantidas em contato com outras de aves como patos e galinhas- d'angola (Santos et al., 2011). As informações obtidas nesses levantamentos corroboram os resultados do presente estudo quanto à ocorrência de L. caponis em granjas avícolas de postura de Minas Gerais. Tais achados sugerem que esta espécie, a princípio, seja pouco frequente nos plantéis avícolas comerciais no Brasil, sendo mais frequente em criações de subsistência, o que concorda com os achados do presente estudo. Outras espécies, tais como as do gênero Menacanthus são mais prevalentes e importantes economicamente (Figueiredo et al., 1993). Embora a ocorrência observada seja baixa, o estudo de aspectos epidemiológicos das infestações por este ectoparasito é necessário visando à prevenção do aumento de sua ocorrência e para que se possam adotar medidas de controle mais efetivas em granjas onde a sua presença já foi detectada.

Em relação à verificação dos aspectos epidemiológicos da ocorrência de $L$. caponis em estabelecimentos comerciais avícolas de postura no estado de Minas Gerais, são apresentadas na Tabela 1 as características que foram selecionadas nos procedimentos de triagem. As variáveis incluídas no modelo de regressão de Poisson destinado à verificação dos principais fatores predisponentes para a ocorrência de $L$. caponis no estado de Minas Gerais são apresentadas na Tabela 2.

Tabela 1: Características potencialmente associadas à ocorrência de infestações por Lipeurus caponis em estabelecimentos avícolas de postura no estado de Minas Gerais, no ano de 2012, selecionadas, na triagem com testes estatísticos univariados $(p<0,15)$

\begin{tabular}{|c|c|}
\hline Característica & Valor $p$ \\
\hline Região* & 0.000 \\
\hline $\begin{array}{c}\text { Tipo de instalação } \\
\text { (gaiola, piso com cama ou piso sem cama) * }\end{array}$ & 0.060 \\
\hline Cidade de localização da granja* & 0.000 \\
\hline Linhagem predominante no galpão* & 0.000 \\
\hline Número de galinhas por granja\# & 0.058 \\
\hline Número de galinhas por gaiola* & 0.013 \\
\hline Faixa de galinhas por metro quadrado* & 0.095 \\
\hline Presença de criações de subsistência de galinhas domésticas ${ }^{*}$ & 0.006 \\
\hline Uso de lança-chamas ("Vassoura de fogo")" & 0.010 \\
\hline Uso de óleo mineral visando o combate de ectoparasitos* & 0.031 \\
\hline Presença de Ornithonyssus sylviarum ${ }^{*}$ & 0.072 \\
\hline Presença de Menacanthus stramineus* & 0.004 \\
\hline
\end{tabular}

Tabela 2: Características predisponentes para ocorrência de Lipeurus caponis em galpões de granjas avícolas comerciais de poedeiras do Estado de Minas Gerais, Brasil, 2012

\begin{tabular}{|c|c|c|c|c|}
\hline Característica & $\begin{array}{c}\text { Razão de } \\
\text { Prevalência }\end{array}$ & \multicolumn{2}{|c|}{$\begin{array}{l}\text { Intervalo de Confiança } \\
(95 \%)\end{array}$} & Valor $p$ \\
\hline $\begin{array}{c}\text { Tipo de instalação } \\
\text { (gaiola, }{ }^{*} \text { piso com cama, piso sem cama) }\end{array}$ & 51,56 & 1,73 & 1528,29 & 0,023 \\
\hline $\begin{array}{c}\text { Presença de criações de subsistência de galinhas } \\
\text { domésticas nas adjacências }\end{array}$ & 106,89 & 3,16 & 3606,83 & 0,009 \\
\hline Linhagem predominante no galpão & 1,8 & 1,16 & 2,96 & 0,010 \\
\hline
\end{tabular}

Número de observações válidas: 429

*característica de referência para elaboração das Razões de Prevalência.

Valor P no Teste de qui-quadrado de Pearson para qualidade de ajuste do modelo: 1,000.

As variáveis região $(p=0,450)$ e cidade $(p=0,586)$ não permaneceram nos modelos de regressão em função da perda 
de significância. Logo, é provável que a distribuição geográfica dos estabelecimentos avícolas não seja determinante para a ocorrência dos mesmos.

Características associadas à densidade dos lotes, a exemplo do número total de galinhas por granja $(p=0,430)$, o número de galinhas por gaiola $(p=0,335)$ e a faixa de galinhas por metro quadrado $(p=0,998)$ não apresentaram relação com a ocorrência de $L$. caponis. Entretanto, em um estudo realizado em setenta estabelecimentos avícolas no Paquistão, observou-se maior ocorrência de malófagos em aves mantidas em criações com altas densidades de aves (Nadeem et al., 2007). Esse estudo verificou que galinhas mantidas em locais com disponibilidade de espaço variando de 0,43 a $0,55 \mathrm{~m}^{2}$ apresentaram maiores infestações quando comparadas àquelas mantidas em área com uma ave a cada $0,61 \mathrm{~m}^{2}$. Este mesmo estudo também verificou maior ocorrência de malófagos em galinhas mais velhas, com idade estimada entre 36 e 72 semanas e em criações sobre o piso. Tais informações, entretanto, são discordantes dos resultados obtidos no presente estudo, uma vez que não foi observada relação entre a idade das aves e a ocorrência desta espécie. Possivelmente, essa discordância seja decorrente das características de análise dos dados, uma vez que o presente trabalho analisou separadamente os efeitos de cada variável, assim como suas interações, e o trabalho realizado no Paquistão executou tal procedimento sem discriminar os efeitos de ambas as características.

O uso de óleo mineral $(p=0,117)$, estratégia empregada para o combate de ácaros hematófagos em aviários de postura (Guimarães e Tucci, 1992; Rezende et al., 2013), não apresentou relação com a ocorrência de $L$. caponis. Logo, presume-se que os mecanismos de ação pesticida destas substâncias não sejam eficazes contra $L$. caponis. Portanto, para a confirmação desta hipótese demanda-se a realização de estudos mais específicos. Também não foi verificada a associação entre a ausência deste parasito em galpões e o uso de lança-chamas ("vassoura de fogo") $(p=0,995)$ nas instalações. Este resultado se deve provavelmente às características biológicas deste artrópode, uma vez que fitirápteros do gênero Lipeurus são parasitos permanentes de porções basais e cobertas das penas, onde se reproduzem. Assim, tais invertebrados, ao contrário de outras espécies, tal como o ácaro hematófago Dermanyssus gallinae, não são comumente encontrados sobre as instalações das granjas avícolas, as quais constituem o alvo de ação do lançachamas (Arora e Chopra, 1957; Guimarães et al., 2001). Logo, intervenções sobre locais que não a superfície corporal das aves, como é o caso do uso de lança-chamas, podem não ter impacto significativo sobre as populações de $L$. caponis. Além disso, o uso de vassoura de fogo nos galpões de criação avícola é realizado no fim do ciclo de produção avícola, o que poderia propiciar tempo suficiente para a recuperação das populações de ectoparasitos que eventualmente venham a ser afetadas.

Não foi observada relação entre a presença de outros ectoparasitos nos galpões, como Ornithonyssus sylviarum (Acari: Macronyssidae) (Canestrini e Fanzago, 1877) $(p=0,995)$ e Menacanthus stramineus (Phithiraptera: Menoponidae) (Nitzsch, 1818) ( $p=0,262)$, e a ocorrência de $L$. caponis. Assim, sugere-se que tais espécies, embora compartilhem o mesmo hospedeiro, não estabelecem entre si relação de competição que possa impedir o estabelecimento de uma ou de ambas. Entretanto, para a comprovação desta hipótese deve-se realizar um estudo da dinâmica populacional das espécies em hospedeiros comuns. Em tais estudos deve ocorrer uma quantificação dos níveis de infestação e tamanho das populações de cada espécie, haja vista o fato de que, caso ocorra competição entre tais espécies, não há impedimento da ocorrência de coinfestações, mas pode haver interferência na magnitude das populações das espécies.

A presença de aves sinantrópicas nas adjacências dos galpões, com exceção de criações de subsistência de galinhas domésticas, não apresentou significância tanto em testes estatísticos uni quanto em multivariados, o que reitera que $L$. caponis realmente seja um parasito específico de galiformes, tal como indicado em outro estudo (Emerson, 1956). Devido aos valores do intervalo de confiança das IRR obtidos, verificouse que a presença de criações de subsistência de galinhas domésticas nos arredores de galpões provoca um incremento nas chances de ocorrência de infestações por $L$. caponis (Tabela 2). As criações de subsistência são aquelas nas quais as aves são mantidas em pequeno número para consumo próprio dos residentes na propriedade e sem finalidade comercial. Tais aves muitas vezes são mantidas de forma negligente nas proximidades e dentro dos estabelecimentos avícolas comerciais e são alvo de escassas medidas sanitárias. Assim, elas podem ser reservatórios e fontes de parasitos para as aves destinadas à exploração comercial.

O tipo de instalação dos galpões também constituiu um fator de risco para a ocorrência de $L$. caponis (Tabela 2 ). De acordo com os resultados obtidos neste estudo, observou-se que a ocorrência deste parasito foi maior em criações sobre o piso do que em gaiolas. Provavelmente, estes resultados foram influenciados pela ausência de dispositivos de remoção mecânica do esterco e dos ovos. Nos estabelecimentos em que este dispositivo era utilizado não houve a ocorrência de L. caponis. Estes resultados concordam com outros estudos que observaram a ocorrência desta espécie somente em aves mantidas sobre o solo (Figueiredo et al., 1993; Santos-Prezoto et al., 2003).

A linhagem genética predominante nos galpões também foi considerada um fator de risco para a ocorrência de $L$. caponis. Essa característica obteve Razão de Prevalência igual a 1,8 (Tabela 2), sugerindo que o risco de ocorrência do parasito aumenta no caso de algumas linhagens. Tais diferenças observadas entre as linhagens podem advir, por exemplo, de diferentes condições do sistema imunológico em função de variações genéticas das galinhas. Outro estudo também constatou a diferença entre certas linhagens de aves quanto à resistência a ectoparasitos (Owen et al., 2008).

\section{Conclusão}

A ocorrência de $L$. caponis em granjas avícolas comerciais de postura no estado de Minas Gerais foi relativamente baixa quando comparada com outros fitirápteros avaliados em outros estudos. A presença de galinhas de subsistência, bem como a linhagem genética e o tipo de instalação, foram principais fatores de risco que incrementaram consideravelmente as chances de ocorrência desse parasito em aviários. 


\section{Agradecimentos}

Os autores agradecem ao Instituto Mineiro de Agropecuária (IMA) e proprietários dos estabelecimentos avícolas pelas autorizações de visitação. Agradece-se também ao CNPq (Conselho Nacional de Desenvolvimento Científico e Tecnológico), FAPEMIG (Fundação de Amparo à Pesquisa do Estado de Minas Gerais) e CAPES (Coordenação de Aperfeiçoamento de Pessoal de Nível Superior) pelo apoio financeiro e bolsas de estudo.

\section{Referências}

ARORA, G.L.; CHOPRA, N.P. Some observations on the biology of Lipeurus tropicalis Peters (Mallophaga: Ischnocera). Research Bulletin of Panjab University, n.130, p. 485-491, 1957.

CUNHA, L.M. Aspectos epidemiológicos relacionados à ocorrência de ácaros hematófagos em granjas comerciais de postura no estado de Minas Gerais e avaliação de armadilhas para captura de Dermanyssus gallinae (Acari: Dermanyssidae) (De Geer, 1778). 2013. 48 f. Tese (Doutorado) - Escola de Veterinária - Universidade Federal de Minas Gerais, Belo Horizonte, 2013.

DOHOO, I.; MARTIN, W.; STRYHN, H. Veterinary epidemiologic research. Charlottetown, Canadá: Atlantic Veterinary College, 2003, 706 p.

EMERSON, K.C. Mallophaga (chewing lice) occurring on the domestic chicken. Journal of Kansas Entomological Society, v. 29, n. 2, p. 63-79, 1956.

FIGUEIREDO, S.M.; GUIMARÃES, J.H.; GAMA, N.M.S.Q. Biologia e Ecologia de Malófagos (Insecta: Phthiraptera) em aves de postura de granjas industriais. Revista Brasileira de Parasitologia Veterinária, v. 2, n.1, p. 45-51, 1993.

GUERRA, R.M.S.N.C.; CHAVES, E.P.; PASSOS, T.M.G.; SANTOS, A.C.G. Species, Dynamics and Population Composition of Phthiraptera in Free-Range Chickens (Gallus gallus L.) in São Luís Island, State of Maranhão. Neotropical Entomology, v. 37, n. 3, p. 259-264, 2008.

GUIMARÃES, J.H.; TUCCI, E.C. Evaluation of mineral oil in control of Dermanyssus gallinae (De Geer, 1778) (Acari, Dermanyssidae) in field and laboratory conditions. Revista Brasileira de Entomologia, v. 36, p. 859-862, 1992.

GUIMARÃES, J.H.; TUCCI, E.C.; BARROS-BATTESTI, D.M. Ectoparasitos de importância veterinária. São Paulo: Plêiade/ FAPESP, 2001, $218 \mathrm{p}$.
KRANTZ, G.W. Collection, Rearing, and Preparation for Study. In: Krantz, G.W. A Manual of Acarology. Corvallis, Oregon: OSU Bookstores, inc., 1978, p.77- 98.

NADEEM, M.; KHAN, M.N.; IQBAL, Z.; SAJID, M.S.; ARSHAD M., YASEEN, M. Determinants influencing prevalence of louse infestations on layers of District Faisalabad (Pakistan). British Poultry Science, v. 48, n. 5, p. 546-550, 2007.

OWEN, J. P.; DELANY M. E.; MULLENS, B. A. MHC haplotype involvement in avian resistance to an ectoparasite. Immunogenetics, v. 60, n. 10, p. 621- 631, 2008.

PRICE, M. A.; GRAHAM, O. H. 1997. Chewing and Sucking lice as parasites of mammals and birds. U.S. Department of Agriculture, Technical Bulletin, n. 1849, 309 p.

REZENDE, L.C.; CUNHA, L.M.; TEIXEIRA, C.M.; OLIVEIRA, P.R.; MARTINS, N.R.S. Mites affecting hen egg production : some considerations for Brazilian farms. Ciencia Rural, v. 43, n. 7, p. 1230-1237, 2013.

SANTOS, A.C.G.; RODRIGUES, A.L.; SANTOS, S.B.; LIMA, R.C.A.; GUERRA, R.M.S.N.C. Phthiraptera (Arthropoda, Insecta) in Gallus gallus from isolated and mixed backyard rearing systems. Revista Brasileira de Parasitologia Veterinária, v. 20, n.1, p. 17-21, 2011.

SANTOS, L.S.S.; SANTOS, L.M.J.F.; AGUIAR, C.L.G.; RUAS, J.L.; FARIAS, N.A.R. Parasitismo de Gallus gallus (Linnaeus, 1758) por espécies de Phthiraptera em criações coloniais na região sul do Rio Grande do Sul, Brasil. Arquivos do Instituto Biológico, v. 80, n. 2, p. 17-221, 2013.

SANTOS-PREZOTO, H.H.; SILVA, M.O.; DAEMON, E.; D’AGOSTO, M.; PREZOTO, F. Sítios de localização de ectoparasitos em Gallus gallus Linnaeus, 1758. Revista Brasileira de Zoociências, v. 5, n. 1, p. 129-135, 2003.

SYCHRA, O.; HARMAT, P.; LITERÁK, I. Chewing lice (Phthiraptera) on chickens (Gallus gallus) from small backyard flocks in the eastern part of the Czech Republic. Veterinary Parasitology, v. 152, n. 3-4, p. 344-348, 2008. 